\title{
Risk-Averse Suppliers' Optimal Pricing Strategies in a Two-Stage Supply Chain
}

\author{
Rui Shen, ${ }^{1}$ Zhiqing Meng, ${ }^{1}$ Xinsheng Xu, ${ }^{1,2}$ and Min Jiang ${ }^{1}$ \\ ${ }^{1}$ College of Economics and Management, Zhejiang University of Technology, Hangzhou, Zhejiang 310032, China \\ ${ }^{2}$ Department of Mathematics and Information Science, Binzhou University, Binzhou, Shandong 256603, China \\ Correspondence should be addressed to Rui Shen; shenruil26@126.com
}

Received 3 April 2013; Revised 21 June 2013; Accepted 27 June 2013

Academic Editor: Tinggui Chen

Copyright (c) 2013 Rui Shen et al. This is an open access article distributed under the Creative Commons Attribution License, which permits unrestricted use, distribution, and reproduction in any medium, provided the original work is properly cited.

\begin{abstract}
Risk-averse suppliers' optimal pricing strategies in two-stage supply chains under competitive environment are discussed. The suppliers in this paper focus more on losses as compared to profits, and they care their long-term relationship with their customers. We introduce for the suppliers a loss function, which covers both current loss and future loss. The optimal wholesale price is solved under situations of risk neutral, risk averse, and a combination of minimizing loss and controlling risk, respectively. Besides, some properties of and relations among these optimal wholesale prices are given as well. A numerical example is given to illustrate the performance of the proposed method.
\end{abstract}

\section{Introduction}

In traditional researches about pricing strategies of suppliers in two-stage supply chains, suppliers were always supposed to take predominant positions in deciding wholesale prices to maximize their profits. However, changes brought by globalization and standardization have forced suppliers to face competition from other suppliers with the same or similar products or services. Therefore suppliers have to allow retailers to negotiate for lower wholesale prices. So in recent years researchers turned their attention to pricing strategies of suppliers under competitive circumstances. For example, Dai et al. [1] studied the pricing strategies of multiple firms providing the same service in competition for a common pool of customers in a revenue management context. Sohn et al. [2] derived a dynamic pricing model and then a pricing policy for a mobile phone manufacturer to maximize his/her profit under the competitive environment. Xiao and Qi [3] developed an adverse selection model for a two-stage supply chain consisting of a manufacturer, a retailer, and a potential outside entrant manufacturer and obtained the optimal decisions for all players. Li et al. [4] investigated two-supplier pricing strategies and derived the sufficient condition of an equilibrium price under the environment where the two suppliers compete with each other. Wang et al. [5] studied the markup pricing strategies in a supply chain with one dominant retailer and multiple competitive manufacturers. To find a mutually beneficial relation, Voeth and Herbst [6] investigated the opportunities for a manufacturer and a retailer to collaborate on pricing, and demonstrated that it is attained in some cases. Arcelus et al. [7] analyzed the manufacturer's optimal wholesale pricing strategy facing a profit-maximizing retailer when the manufacturer possesses the full information about the cost and the functional relationship between demand and price. $\mathrm{Lu}$ et al. [8] highlighted the importance of services from manufacturers in the interactions between two competing manufacturers and one common retailer and proposed a game-theoretic framework for obtaining the equilibrium solutions for each entity. Xia [9] studied the competitive strategies between two coexisting suppliers in a two-echelon supply chain. Pricing strategies for different retailer groups are suggested to the competitive suppliers accordingly. Chen et al. [10] presented a review of the manufacturer's pricing strategies in a two-stage supply chain where the retailer's demand is warranty-period dependent. Sana [11] investigated the issue of channel coordination between a manufacturer and a retailer both facing stochastic demand that is sensitive 
to promotional efforts and provided an analytical method to determine the optimal contract parameters of the channel.

However, in recent years, unpredictable disasters like earthquakes and economic crisis have disrupted the operation of supply chains, which further have also brought great losses to agents in supply chains. So agents in supply chains nowadays pay more attention to minimizing their losses than maximizing the profits, but few research paid attention to this. The suppliers in such environment may become risk averse. They must decide a suitable wholesale price, which is neither very low nor very high. Obviously were it very low, suppliers would suffer losses (called current loss) in profits that were supposed to be earned. Were it very high, retailers may turn to other suppliers, which hurts the suppliers longterm profits and results in future loss. In this paper, we are to find such a suitable wholesale price that takes both the current loss and future loss into consideration. But before doing this, we first introduce the following loss function for suppliers:

$$
L(x)=A(\xi-x)^{+}+B q(x-\xi)^{+} .
$$

Here, $x$ is the wholesale price offered by a supplier; $q$ is the order quantity from a retailer; $\xi$, a random variable, is the market wholesale price for a product; $A$ is the shortage penalty coefficient of the supplier for his/her giving a very low wholesale price, and $B$ is the excess penalty coefficient of the supplier for his/her giving a very high wholesale price. In the right hand of (1), the first item represents the current loss, and the second item represents the future loss. In the current literature, the suppliers are supposed to give such wholesale prices so as to maximize their profits or minimize their costs in a deal, while the influence of these decisions on their future profits or costs is neglected. However, (1) considers the influence of the supplier's decision both on the current profit and the future profit. It implies that the supplier must obtain a balance between the current profit and the future profit in deciding the wholesale price. Then by minimizing the expected loss $E[L(x)]$, adopting the CVaR measure, and combining the previous two, we obtain three different optimal solutions to the wholesale price under different situations, which are risk neutral, risk averse, and a combination of minimizing loss and risk control, respectively. It is easily checked that the optimal wholesale price obtained under the risk neutral situation by minimizing the expected loss $E[L(x)]$, where $E$ is the expectation operator, increases with the growth of the shortage penalty coefficient $A$ and decreases with the excess penalty coefficient $B$ and the order quantity $q$, respectively. Then, by adopting the CVaR measure of risk management, we obtain an optimal wholesale price under the risk-averse situation, which equals the optimal solution to the following problem:

$$
\min _{x \in[c, m]} \operatorname{CVaR}_{\alpha}(x)=E\left[L(x) \mid L(x) \geq \operatorname{VaR}_{\alpha}(x)\right] .
$$

Finally, in order to balance the expected loss and the CVaR loss of the supplier, which implies the supplier considers both the minimization of expected loss and the risk control, we define the following objective function with a combination of the two objectives:

$$
\lambda E[L(x)]+(1-\lambda) \operatorname{CVaR}_{\alpha}(x),
$$

where $\lambda \in[0,1]$ is the weight of the expected loss, which represents the relative importance of the expected loss as compared to the CVaR loss for the supplier, and by minimizing the previous function the optimal wholesale price is obtained.

The rest of this paper is organized as follows. Section 2 introduces the preliminaries about VaR and CVaR. Section 3 studies the optimal decisions of the supplier under different objectives and the properties of these optimal decisions. Section 4 gives some numerical examples, with conclusions given in Section 5.

\section{Some Preliminaries about CVaR}

In this section, we give some preliminaries about VaR and CVaR.

Value-at-Risk (VaR) is a popular risk measure that has achieved high status of being written into industry regulations. For a decision $x$, let $l(x, \xi)$ be the loss associated with $x$ and the random variable $\xi$. The $\alpha$-VaR with $x$ is defined as

$$
\operatorname{VaR}_{\alpha}(x)=\min \{y \in R \mid \operatorname{Pr}\{l(x, \xi) \leq y\} \geq \alpha\},
$$

where $\operatorname{Pr}\{l(x, \xi) \leq y\}$ denotes the probability of $l(x, \xi)$ not exceeding the value $y$. The value of $\operatorname{VaR}_{\alpha}(x)$ represents the minimal loss of decision $x$ with the confidence level $\alpha$. Artzner et al. [12] and Mauser and Rosen [13] pointed out that VaR has some undesirable mathematical characteristics such as nonsubadditivity and nonconvexity, which always hinders its efficient usage. Therefore, Rockafellar and Uryasev [14] and Rockafellar and Uryasev [15] introduced another risk measure: Conditional Value-at-Risk (CVaR), which is defined as the expected value of loss exceeding the $\operatorname{VaR}_{\alpha}(x)$. CVaR has some attractive properties such as coherence and convexity, which makes it widely used in risk management as compared to $\mathrm{VaR}$. The CVaR of $x$ with a confidence level $\alpha$ can be defined as

$$
\begin{aligned}
\operatorname{CVaR}_{\alpha}(x) & =E\left[l(x, \xi) \mid l(x, \xi) \geq \operatorname{VaR}_{\alpha}(x)\right] \\
& =\frac{1}{1-\alpha} \int_{l(x, z) \geq \operatorname{VaR}_{\alpha}(x)} l(x, z) \phi(z) d z,
\end{aligned}
$$

where $\phi(z)$ is the probability density function of $\xi$, and $\operatorname{VaR}_{\alpha}(x)$ is defined by (4). To compute, Rockafellar and Uryasev [14] introduced the function of $F(x, u)$ :

$$
F(x, u)=u+\frac{1}{1-\alpha} E\left[(l(x, \xi)-u)^{+}\right],
$$

and proved that the minimum of $\operatorname{CVaR}(x)$ can be obtained by minimizing the function $F(x, u)$, and the corresponding $\operatorname{VaR}_{\alpha}(x)$ can be reached simultaneously in this way.

\section{Main Results}

For suppliers, it is supposed that the market wholesale price $\xi$ is a random variable, and let $f(\cdot)$ and $F(\cdot)$ be its probability density function and cumulative distribution function, respectively. Without loss of generality, it is assumed that the 
inverse of $F(\cdot)$ exists, and $\xi \in[c, m]$ where $0<c<m$. For a given order quantity $q$ from a retailer, the loss $L(x)$ of the supplier is given by (1). Now, let us discuss the optimal decisions of the supplier under the previous three different situations as to loss $L(x)$.

3.1. Optimal Wholesale Price in Minimizing Expected Loss. Evidently, to find the optimal wholesale price that minimizes the expected loss of the supplier, we need to obtain the optimal solution to the following problem:

$$
\min _{x \in[c, m]} E[L(x)]
$$

Then, we have the following result about $(\mathrm{P})$.

Theorem 1. For the supplier, the optimal solution to $(P)$ is given by

$$
x_{0}^{*}=F^{-1}\left(\frac{A}{A+B q}\right) .
$$

Proof. For a wholesale price $x$ given by the supplier, we have

$$
L(x)=A(\xi-x)^{+}+B q(x-\xi)^{+} .
$$

It follows from $(\xi-x)^{+}=(\xi-x)+(x-\xi)^{+}$that

$$
L(x)=A \xi-A x+(A+B q)(x-\xi)^{+} .
$$

Then the expected loss $\mu(x)=E[L(x)]$ is given by

$$
\mu(x)=A(E[\xi]-x)+(A+B q) \int_{c}^{x}(x-t) d F(t),
$$

which implies

$$
\mu^{\prime}(x)=(A+B q) F(x)-A .
$$

Then the solution to problem (P) is obtained by solving the $\mu^{\prime}(x)=0$ which gives

$$
x_{0}^{*}=F^{-1}\left(\frac{A}{A+B q}\right) \text {. }
$$

Corollary 2. For the supplier, the optimal wholesale price $x_{0}^{*}$ decreases with the growth of the excess penalty coefficient $B$.

In fact, if the excess penalty coefficient B improves, which implies that the supplier pays more attention to the long-term cooperation with the retailer, the supplier will decrease the wholesale price to maintain the cooperation with the retailer in the future.

Corollary 3. For the supplier, the optimal wholesale price $x_{0}^{*}$ increases with the growth of shortage penalty coefficient $A$.

In fact, if the shortage penalty coefficient A improves, that is, the supplier pays more attention to the loss of the current transaction and ignores the future one, then he/she will increase the wholesale price to lessen his/her loss in the current transaction.
Corollary 4. For the supplier, the optimal wholesale price $x_{0}^{*}$ decreases with the growth of the order quantity $q$ from the retailer.

By Corollary 4, the wholesale price will decrease with the growth of the order quantity $q$ from the retailer.

3.2. Optimal Wholesale Price in Minimizing CVaR Loss. In the above subsection, we discuss how to decide the optimal wholesale price that minimizes the expected loss of the supplier, but this approach may lead to an unpredictable large loss since risks are not considered. To control risks that may lead to possible losses for the supplier, we now consider minimizing the CVaR loss, which can determine the minimal loss of the supplier for a given confidence level.

For the confidence level $\alpha$, the $\alpha$-VaR of the supplier with respect to the wholesale price $x$ is given by

$$
\operatorname{VaR}_{\alpha}(x)=\min \{y \in R \mid \operatorname{Pr}\{L(x) \leq y\} \geq \alpha\},
$$

where $\operatorname{Pr}\{L(x) \leq y\}$ denotes the probability of $L(x)$ not exceeding the value $y$. Then the CVaR loss of the supplier about $x$ can be defined as

$$
\mathrm{CVaR}_{\alpha}(x)=E\left[L(x) \mid L(x) \geq \operatorname{VaR}_{\alpha}(x)\right] .
$$

Evidently, we need to find the optimal solution to the following problem:

$$
\min _{x \in[c, m]} \mathrm{CVaR}_{\alpha}(x)
$$

Then we have the following result.

Theorem 5. For the risk-averse supplier, the optimal solution to $\left(P_{1}\right)$ is given by

$$
x_{1}^{*}=\frac{1}{A+B q}\left[A F^{-1}\left(\frac{A+B q \alpha}{A+B q}\right)+B q F^{-1}\left(\frac{A(1-\alpha)}{A+B q}\right)\right] \text {. }
$$

Proof. By (9), we have $L(x)=A \xi-A x+(A+B q)(x-\xi)^{+}$. Now, we define a convex function

$$
\begin{aligned}
& h(x, v) \\
& =v+\frac{1}{1-\alpha} E[L(x)-v]^{+} \\
& =v+\frac{1}{1-\alpha} \int_{c}^{m}\left[A t-A x+(A+B q)(x-t)^{+}-v\right]^{+} d F(t) \\
& =v+\frac{1}{1-\alpha} \int_{c}^{x}[B q x-B q t-v]^{+} d F(t) \\
& \quad+\frac{1}{1-\alpha} \int_{x}^{m}[A t-A x-v]^{+} d F(t) .
\end{aligned}
$$

Based on the result in Section 2, the optimal solution to $\left(\mathrm{P}_{1}\right)$ equals the optimal solution to the following problem:

$$
\min _{x \in[c, m]}\left[\min _{v \in R} h(x, v)\right] .
$$


Then, for any fixed $x$, we distinguish the following different cases.

(i) $v \geq B q x$.

In this case, by (16), we have

$$
\begin{aligned}
& h(x, v) \\
& =v+\frac{1}{1-\alpha} \int_{c}^{x} 0 d F(t) \\
& \quad+\frac{1}{1-\alpha} \int_{x+(v / A)}^{m}[A t-A x-v] d F(t), \\
& \frac{\partial h(x, v)}{\partial v}=1-\frac{1}{1-\alpha}\left[1-F\left(x+\frac{v}{A}\right)\right] .
\end{aligned}
$$

Obviously, when $v$ is sufficiently large $(v \geq A(m-x))$, it follows from (19) that $\partial h(x, v) / \partial x=1>0$ holds. Then if it satisfies $\left.(\partial h(x, v) / \partial x)\right|_{v=B q x}=1-(1 /(1-\alpha))[1-F(x+$ $(B q x / A))]<0$, which implies $x<(A /(A+B q)) F^{-1}(\alpha)$, it follows from (19) and $\partial h(x, v) / \partial v=0$ that the optimal solution $v^{*}$ to $\min _{v \in R} h(x, v)$ solves

$$
\frac{\partial h(x, v)}{\partial v}=1-\frac{1}{1-\alpha}\left[1-F\left(x+\frac{v}{A}\right)\right]=0 .
$$

That is

$$
v^{*}=A\left[F^{-1}(\alpha)-x\right]
$$

(ii) $0<v<B q x$.

In this case, by (16), we have

$$
\begin{aligned}
h(x, v)= & v+\frac{1}{1-\alpha} \int_{c}^{x-(v / B q)}[B q x-B q t-v] d F(t) \\
& +\frac{1}{1-\alpha} \int_{x+(v / A)}^{m}[A t-A x-v] d F(t), \\
\frac{\partial h(x, v)}{\partial v}= & 1-\frac{1}{1-\alpha}\left[1+F\left(x-\frac{v}{B q}\right)-F\left(x+\frac{v}{A}\right)\right] .
\end{aligned}
$$

Obviously, it satisfies

$$
\left.\frac{\partial h(x, v)}{\partial v}\right|_{v=0}=1-\frac{1}{1-\alpha}<0
$$

Then if it satisfies $\left.(\partial h(x, v) / \partial x)\right|_{v=B q x}=1-(1 /(1-\alpha))[1-$ $F(x+(B q x / A))] \geq 0$, which implies $x \geq(A /(A+B q)) F^{-1}(\alpha)$, it follows from $(23)$ and $\partial h(x, v) / \partial v=0$ that the optimal solution $v^{*}$ to $\min _{v \in R} h(x, v)$ solves

$$
\frac{\partial h(x, v)}{\partial v}=1-\frac{1}{1-\alpha}\left[1+F\left(x-\frac{v}{B q}\right)-F\left(x+\frac{v}{A}\right)\right]=0 .
$$

(iii) $v \leq 0$.

In this case, by (16), we have

$$
\begin{aligned}
h(x, v)= & v+\frac{1}{1-\alpha} \int_{c}^{x}[B q x-B q t-v] d F(t) \\
& +\frac{1}{1-\alpha} \int_{x}^{m}[A t-A x-v] d F(t), \\
& \frac{\partial h(x, v)}{\partial v}=1-\frac{1}{1-\alpha}<0 .
\end{aligned}
$$

Based on the previous analysis, it is clear that for any fixed $x, h(x, v)$ attains minimum when $v>0$. Further, for any fixed $x$, the optimal solution to $\min _{v \in R} h(x, v)$ is given by

$$
v^{*}= \begin{cases}A\left[F^{-1}(\alpha)-x\right] & x<\frac{A}{A+B q} F^{-1}(\alpha), \\ v^{1} & x \geq \frac{A}{A+B q} F^{-1}(\alpha),\end{cases}
$$

where $v^{1}$ is given by (25).

Thus, to solve the problem $\min _{x \in[c, m]}\left[\min _{v \in R} h(x, v)\right]=$ $\min _{x \in[c, m]}\left[h\left(x, v^{*}\right)\right]$, we distinguish the following cases.

(a) $x<(A /(A+B q)) F^{-1}(\alpha)$.

In this case, it follows from (27) that the optimal solution to the problem $\min _{v \in R} h(x, v)$ is given by

$$
v^{*}=A\left[F^{-1}(\alpha)-x\right] \text {. }
$$

Then by (16), we have

$$
\begin{aligned}
h\left(x, v^{*}\right)= & A\left[F^{-1}(\alpha)-x\right]+\frac{1}{1-\alpha} \int_{c}^{x} 0 d F(t) \\
+ & \frac{1}{1-\alpha} \int_{F^{-1}(\alpha)}^{m} A\left(t-F^{-1}(\alpha)\right) d F(t), \\
& \frac{\partial h\left(x, v^{*}\right)}{\partial x}=-A<0 .
\end{aligned}
$$

(b) $x \geq(A /(A+B q)) F^{-1}(\alpha)$.

In this case, it follows from (27) that the optimal solution to the problem $\min _{v \in R} h(x, v)$ is given by $v^{*}=v^{1}$, where $v^{1}$ satisfies (25). By (25), we have

$$
F\left(x+\frac{v^{1}}{A}\right)=F\left(x-\frac{v^{1}}{B q}\right)+\alpha .
$$


Then by (16), we have

$$
\begin{aligned}
h\left(x, v^{1}\right)= & v^{1}+\frac{1}{1-\alpha} \int_{c}^{x-\left(v^{1} / B q\right)}\left[B q x-B q t-v^{1}\right] d F(t) \\
& +\frac{1}{1-\alpha} \int_{x+\left(v^{1} / A\right)}^{m}\left[A t-A x-v^{1}\right] d F(t) \\
& \frac{\partial h\left(x, v^{1}\right)}{\partial x} \\
& =\frac{1}{1-\alpha}\left[B q F\left(x-\frac{v^{1}}{B q}\right)-A\left(1-F\left(x+\frac{v^{1}}{A}\right)\right)\right]
\end{aligned}
$$

It follows from (32) and $\partial h\left(x, v^{1}\right) / \partial x=0$ that the optimal solution $x_{1}^{*}$ to $\min _{x \in[c, m]}\left[h\left(x, v^{*}\right)\right]$ solves

$$
\frac{1}{1-\alpha}\left[B q F\left(x-\frac{v^{1}}{B q}\right)-A\left(1-F\left(x+\frac{v^{1}}{A}\right)\right)\right]=0 .
$$

Then it follows from (30) and (33) that

$$
x_{1}^{*}=\frac{1}{A+B q}\left[A F^{-1}\left(\frac{A+B q \alpha}{A+B q}\right)+B q F^{-1}\left(\frac{A(1-\alpha)}{A+B q}\right)\right] \text {. }
$$

It is easily checked that the optimal solution to $\left(\mathrm{P}_{1}\right)$ is more complicated than that of $(\mathrm{P})$. Similar to Corollaries 2-4, we have the following results.

Corollary 6. For the risk-averse supplier, the optimal solution $x_{1}^{*}$ to $\left(P_{1}\right)$ decreases with the growth of the excess penalty coefficient $B$.

Proof. By Theorem 5, the optimal solution to $\left(\mathrm{P}_{1}\right)$ is given by

$$
x_{1}^{*}=\frac{1}{A+B q}\left[A F^{-1}\left(\frac{A+B q \alpha}{A+B q}\right)+B q F^{-1}\left(\frac{A(1-\alpha)}{A+B q}\right)\right] \text {. }
$$

For simplicity, we denote

$$
\frac{A+B q \alpha}{A+B q}=M, \quad \frac{A(1-\alpha)}{A+B q}=N .
$$

It is obvious that

$$
M-N=\alpha \geq 0
$$

which implies

$$
M \geq N
$$

Thus we have

$$
\begin{aligned}
& \frac{\partial x_{1}^{*}}{\partial B}= \frac{\left(\left(\partial\left[A F^{-1}(M)+B q F^{-1}(N)\right]\right) /(\partial B)\right)(A+B q)}{(A+B q)^{2}} \\
&-\frac{\left[A F^{-1}(M)+B q F^{-1}(N)\right] q}{(A+B q)^{2}} \\
&=\left(\left(-A q(1-\alpha)\left[A\left(f\left(F^{-1}(M)\right)\right)^{-1}\right.\right.\right. \\
&\left.\left.\left.+B q\left(f\left(F^{-1}(N)\right)\right)^{-1}\right]\right) \times(A+B q)^{-1}\right) \\
&-\frac{\left[A F^{-1}(M)+B q F^{-1}(N)\right] q}{(A+B q)^{2}} \\
&-\frac{\left.A q+B q) q F^{-1}(N)\right)\left((A+B q)^{2}\right)^{-1}}{(A+B q)^{2}\left[F^{-1}(M)-F^{-1}(N)\right] .} \\
&=-\left(A q(1-\alpha)\left[A\left[f\left(F^{-1}(M)\right)\right]^{-1}\right]\right. \\
&\left.+B q\left[f\left(F^{-1}(N)\right)\right]^{-1}\right) \times\left((A+B q)^{3}\right)^{-1}
\end{aligned}
$$

It follows from $M \geq N$ that $F^{-1}(M)-F^{-1}(N) \geq 0$ holds, which by (39) implies $\partial x_{1}^{*} / \partial B \leq 0$, and $x_{1}^{*}$ decreases with the growth of the excess penalty coefficient $B$.

Corollary 7. For the risk-averse supplier, the optimal solution $x_{1}^{*}$ to $\left(P_{1}\right)$ increases with the growth of shortage penalty coefficient $A$.

Proof. By Theorem 5, we have

$$
\begin{gathered}
\frac{\partial x_{1}^{*}}{\partial A}=\frac{\left(\left(\partial\left[A F^{-1}(M)+B q F^{-1}(N)\right]\right) /(\partial A)\right)(A+B q)}{(A+B q)^{2}} \\
-\frac{\left[A F^{-1}(M)+B q F^{-1}(N)\right]}{(A+B q)^{2}} \\
=\left(\left(\left(B q(1-\alpha)\left[A\left(f\left(F^{-1}(M)\right)\right)^{-1}\right]\right.\right.\right. \\
\left.\left.+B q\left(f\left(F^{-1}(N)\right)\right)^{-1}\right)(A+B q)^{-1}\right) \\
\left.+(A+B q) F^{-1}(M)\right) \times\left((A+B q)^{2}\right)^{-1}
\end{gathered}
$$$$
-\frac{\left[A F^{-1}(M)+B q F^{-1}(N)\right] q}{(A+B q)^{2}}
$$ 


$$
\begin{aligned}
= & \frac{B q(1-\alpha)\left[A\left(f\left(F^{-1}(M)\right)\right)^{-1}+B q\left(f\left(F^{-1}(N)\right)\right)^{-1}\right]}{(A+B q)^{3}} \\
& +\frac{B q}{(A+B q)^{2}}\left[F^{-1}(M)-F^{-1}(N)\right] .
\end{aligned}
$$

It follows from $M \geq N$ that $F^{-1}(M)-F^{-1}(N) \geq 0$ holds. Then it concludes from (40) that $\partial x_{1}^{*} / \partial A \geq 0$, which implies that the optimal solution $x_{1}^{*}$ to $\left(\mathrm{P}_{1}\right)$ increases with the growth of shortage penalty coefficient $A$.

Corollary 8. For the risk-averse supplier, the optimal solution $x_{1}^{*}$ to $\left(P_{1}\right)$ decreases with the growth of the order quantity $q$ from the retailer.

Proof. By Theorem 5, we have

$$
\begin{aligned}
\frac{\partial x_{1}^{*}}{\partial q}= & \frac{\left(\left(\partial\left[A F^{-1}(M)+B q F^{-1}(N)\right]\right) /(\partial q)\right)(A+B q)}{(A+B q)^{2}} \\
& -\frac{B\left[A F^{-1}(M)+B q F^{-1}(N)\right]}{(A+B q)^{2}} \\
= & (((A B(1-\alpha) \\
& \left.\times\left[A f\left(F^{-1}(M)\right)+B q\left(f\left(F^{-1}(N)\right)\right)^{-1}\right]\right) \\
& \times\left((A+B q)^{2}\right)^{-1} \\
& -\frac{B\left[A F^{-1}(M)+B q F^{-1}(N)\right]}{(A+B q)^{2}} \\
= & -\frac{A B(1-\alpha)\left[A\left[f\left(F^{-1}(M)\right)\right]^{-1}+B q\left[f\left(F^{-1}(N)\right)\right]^{-1}\right]}{(A+B q)^{3}} \\
& -\frac{A B}{(A+B q)^{2}\left[F^{-1}(M)-F^{-1}(N)\right] .}
\end{aligned}
$$

It follows from $M \geq N$ that $F^{-1}(M)-F^{-1}(N) \geq 0$ holds. Then it concludes from (41) that $\partial x_{1}^{*} / \partial q \leq 0$, which implies that the optimal solution $x_{1}^{*}$ to $\left(\mathrm{P}_{1}\right)$ decreases with the growth of the order quantity $q$ from the retailer.

By Corollaries 6-8, the optimal wholesale price that minimizes $\mathrm{CVaR}$ loss decreases with the growth of the excess penalty coefficient $B$ and the order quantity $q$ from the retailer and increases with the growth of shortage penalty coefficient A.
Remark 9. For $\alpha \in(0,1), x_{1}^{*}$ may not be monotone with $\alpha$. By Theorem 5, the optimal solution to $\left(\mathrm{P}_{1}\right)$ is given by

$$
x_{1}^{*}=\frac{1}{A+B q}\left[A F^{-1}\left(\frac{A+B q \alpha}{A+B q}\right)+B q F^{-1}\left(\frac{A(1-\alpha)}{A+B q}\right)\right] \text {. }
$$

Thus we have

$$
\begin{aligned}
\frac{\partial x_{1}^{*}}{\partial \alpha}= & \frac{A B q}{(A+B q)^{2} f\left[F^{-1}(M)\right] f\left[F^{-1}(N)\right]} \\
& \times\left[f\left(F^{-1}(N)\right)-f\left(F^{-1}(M)\right)\right] .
\end{aligned}
$$

Obviously, the previous inequality shows that the sign of $\partial x_{1}^{*} / \partial \alpha$ is the same as that of $f\left(F^{-1}(N)\right)-f\left(F^{-1}(M)\right)$, which maybe positive or negative. Specially, if $f(\cdot)$ increases monotonically, it concludes from $M \geq N$ that $f\left[F^{-1}(M)\right] \geq$ $f\left[F^{-1}(N)\right]$, which implies $\partial x_{1}^{*} / \partial \alpha \leq 0$, and then the optimal wholesale price $x_{1}^{*}$ decreases with the growth of $\alpha$. Otherwise, if $f(\cdot)$ decreases monotonically, it concludes from $M \geq N$ that $f\left[F^{-1}(M)\right] \leq f\left[F^{-1}(N)\right]$, which implies $\partial x_{1}^{*} / \partial \alpha \geq 0$, and then the optimal wholesale price $x_{1}^{*}$ increases with the growth of $\alpha$.

Example 10. For a two-stage supply chain, suppose that the market wholesale price $\xi$ subjects to exponential distribution $e(0.25)$, uniform distribution $U(3,5)$, and normal distribution $N\left(4,0.5^{2}\right)$, respectively. Let $A=100, B=2$, and $q=100$. For different confidence level $\alpha$, we compute the optimal wholesale price of the risk-averse supplier with CVaR measure of loss, with the results listed in Table 1.

As shown in Table 1, if the market wholesale price $\xi$ subjects to the exponential distribution $e(0.25)$, then the optimal wholesale price $x_{1}^{*}$ of the supplier increases with the growth of $\alpha$; if the market wholesale price $\xi$ subjects to the uniform distribution $U(3,5)$, then the optimal wholesale price $x_{1}^{*}$ of the supplier stays the same; if the market wholesale price $\xi$ subjects to the normal distribution $N\left(4,0.5^{2}\right)$, then the optimal wholesale price $x_{1}^{*}$ of the supplier decreases with the growth of $\alpha$.

3.3. Optimal Wholesale Price in Balancing Expected Loss and CVaR Loss. Evidently, the CVaR approach is too conservative for some suppliers, who pay great attention to the loss above the VaR while the part below the VaR is ignored. Therefore, we intend to find a wholesale price that balances the expected loss and CVaR loss. For this aim, we propose the following problem:

$$
\min _{x \in[c, m]}\left[\lambda E(L(x))+(1-\lambda) \operatorname{CVaR}_{\alpha}(x)\right],
$$

where $\lambda \in[0,1]$ is the weight of the expected loss, which represents the relative importance of the expected loss as compared to the CVaR loss. This utility function reflects the fact that suppliers concern both minimization of expected loss and risk control. Then our objective is to find the optimal solution to Problem $\left(\mathrm{P}_{2}\right)$. 
TABLE 1: Optimal wholesale prices for different distributions of $\xi$.

\begin{tabular}{lcccccccccc}
\hline & $\alpha$ & 0.1 & 0.2 & 0.3 & 0.4 & 0.5 & 0.6 & 0.7 & 0.8 & 0.9 \\
\hline$\xi \sim e(0.25)$ & $q_{1}^{*}$ & 1.632 & 1.665 & 1.725 & 1.817 & 1.951 & 2.144 & 2.427 & 2.781 & 3.701 \\
$\xi \sim U(3,5)$ & $q_{1}^{*}$ & 3.667 & 3.667 & 3.667 & 3.667 & 3.667 & 3.667 & 3.667 & 3.667 & 3.667 \\
$\xi \sim N\left(4,0.5^{2}\right)$ & $q_{1}^{*}$ & 3.783 & 3.778 & 3.771 & 3.761 & 3.749 & 3.734 & 3.713 & 3.685 & 3.639 \\
\hline
\end{tabular}

Theorem 11. For the supplier, if it satisfies

$$
\lambda \geq \frac{A}{(A+B q) F\left[(A /(A+B q)) F^{-1}(\alpha)\right]},
$$

then the optimal solution to $\left(P_{2}\right)$ is given by

$$
x_{2}^{*}=F^{-1}\left[\frac{A}{\lambda(A+B q)}\right] \text {; }
$$

otherwise, the optimal solution to $\left(P_{2}\right)$ solves

$$
\begin{gathered}
x_{2}^{*}=\left(\left[A F^{-1}((\alpha(1-\lambda)(A+B q)+(1-\alpha)\right.\right. \\
\left.\times\left(A-\lambda(A+B q) F\left(x_{2}^{*}\right)\right)\right) \\
\left.\times((1-\lambda)(A+B q))^{-1}\right) \\
\left.\left.+B q F^{-1}\left(\frac{(1-\alpha)\left(A-\lambda(A+B q) F\left(x_{2}^{*}\right)\right)}{(1-\lambda)(A+B q)}\right)\right]\right) \\
\quad \times(A+B q)^{-1} .
\end{gathered}
$$

Proof. Similar to the proof of Theorem 5, let us define a convex function

$$
\begin{aligned}
& k(x, v)=\lambda\left[A E \xi-A x+(A+B q) \int_{c}^{x}(x-t) d F(t)\right] \\
&+(1-\lambda)\left[v+\frac{1}{1-\alpha} \int_{c}^{x}(B q x-B q t-v)^{+} d F(t)\right. \\
&\left.+\frac{1}{1-\alpha} \int_{x}^{m}(A t-A x-v)^{+} d F(t)\right] .
\end{aligned}
$$

Obviously, the optimal solution to $\left(\mathrm{P}_{2}\right)$ equals the optimal solution to the following problem:

$$
\min _{x \in[c, m]}\left[\min _{v \in R} k(x, v)\right] .
$$

Note that the first item in the right hand of (47) has nothing to do with $v$; then for any fixed $x$, it is concluded from the proof of Theorem 5 that the optimal solution to $\min _{v \in R} k(x, v)$ is given by

$$
v^{*}= \begin{cases}A\left[F^{-1}(\alpha)-x\right] & x<\frac{A}{A+B q} F^{-1}(\alpha) \\ v^{1} & x \geq \frac{A}{A+B q} F^{-1}(\alpha)\end{cases}
$$

where $v^{1}$ solves

$$
F\left(x+\frac{v^{1}}{A}\right)=F\left(x-\frac{v^{1}}{B q}\right)+\alpha .
$$

Thus, we solve $\min _{x \in[c, m]}\left[\min _{v \in R} k(x, v)\right]=$ $\min _{x \in[c, m]} k\left(x, v^{*}\right)$ in the following two different cases.

(a) $x<(A /(A+B q)) F^{-1}(\alpha)$.

In this case, it follows from (49) that the optimal solution to the problem $\min _{v} k(x, v)$ is given by

$$
v^{*}=A\left[F^{-1}(\alpha)-x\right]
$$

Then by (47), we have

$$
\begin{aligned}
k\left(x, v^{*}\right)= & \lambda\left[A E \xi-A x+(A+B q) \int_{c}^{x}(x-t) d F(t)\right] \\
& +(1-\lambda)\left[A\left(F^{-1}(\alpha)-x\right)+\frac{1}{1-\alpha} \int_{c}^{x} 0 d F(t)\right. \\
& \left.+\frac{1}{1-\alpha} \int_{F^{-1}(\alpha)}^{m}\left(A\left(t-F^{-1}(\alpha)\right)\right) d F(t)\right],
\end{aligned}
$$

$\frac{\partial k\left(x, v^{*}\right)}{\partial x}=\lambda(A+B q) F(x)-A$.

Obviously, it satisfies

$$
\left.\frac{\partial k\left(x, v^{*}\right)}{\partial x}\right|_{x=c}=-A<0 .
$$

If it satisfies $\left.\left(\partial k\left(x, v^{*}\right) / \partial x\right)\right|_{x=(A /(A+B q)) F^{-1}(\alpha)} \geq 0$, that is, $\lambda \geq$ $A /(A+B q) F\left[(A /(A+B q)) F^{-1}(\alpha)\right]$, then it concludes from (53) and $\partial k\left(x, v^{*}\right) / \partial x=0$ that the optimal solution $x^{*}$ to $\min _{x \in[c, m]} k\left(x, v^{*}\right)$ is given by

$$
x_{2}^{*}=F^{-1}\left[\frac{A}{\lambda(A+B q)}\right] \text {. }
$$

Otherwise, we consider the case of $x \geq(A /(A+B q)) F^{-1}(\alpha)$.

(b) $x \geq(A /(A+B q)) F^{-1}(\alpha)$.

In this case, it follows from (49) that the optimal solution to the problem $\min _{v} k(x, v)$ is given by $v^{*}=v^{1}$. 
Then by (47), we have

$$
\begin{aligned}
& k\left(x, v^{*}\right)= k\left(x, v^{1}\right) \\
&=\lambda\left[A E \xi-A x+(A+B q) \int_{\mathcal{C}}^{x}(x-t) d F(t)\right]+(1-\lambda) \\
& \times\left[v^{1}+\frac{1}{1-\alpha}\right. \\
& \quad \times \int_{c}^{x-\left(v^{1} / B q\right)}\left(B q x-B q t-v^{1}\right) d F(t) \\
&\left.\quad+\frac{1}{1-\alpha} \int_{x+\left(v^{1} / A\right)}^{m}\left(A t-A x-v^{1}\right) d F(t)\right],
\end{aligned}
$$

$$
\begin{aligned}
\frac{\partial k\left(x, v^{1}\right)}{\partial x}= & \lambda(A+B q) F(x)-\lambda A \\
& +\frac{1-\lambda}{1-\alpha}\left[B q F\left(x-\frac{v^{1}}{B q}\right)+A F\left(x+\frac{v^{1}}{A}\right)-A\right] .
\end{aligned}
$$

Obviously, it follows from (50) and (57) that

$$
\begin{aligned}
& \frac{\partial k\left(x, v^{1}\right)}{\partial x} \\
& =\lambda(A+B q) F(x)-\lambda A \\
& \quad+\frac{1-\lambda}{1-\alpha}\left[(A+B q) F\left(x-\frac{v^{1}}{B q}\right)-A(1-\alpha)\right] \\
& =\lambda(A+B q) F(x)-A+\frac{1-\lambda}{1-\alpha}(A+B q) F\left(x-\frac{1-\lambda}{1-\alpha}(A+B q)\right. \\
& \left.\left.=\lambda(A+B q) F(x)-A+\frac{v^{1}}{A}\right)-\alpha\right] . \\
& \quad \times\left[F \left(x+\frac{1}{1-}\right.\right.
\end{aligned}
$$$$
=\lambda(A+B q) F(x)-A+\frac{1-\lambda}{1-\alpha}(A+B q) F\left(x-\frac{v^{1}}{B q}\right)
$$

It follows from $(58)$ and $\partial k\left(x, v^{1}\right) / \partial x=0$ that

$$
v^{1}=B q x-B q F^{-1}\left[\frac{(1-\alpha)(A-\lambda(A+B q) F(x))}{(1-\lambda)(A+B q)}\right] .
$$

Thus, it concludes form (59), (60), and $\partial k\left(x, v^{1}\right) / \partial x=0$ that the optimal solution $x_{2}^{*}$ to $\min _{x \in[c, m]} k\left(x, v^{*}\right)$ solves

$$
\begin{aligned}
& x_{2}^{*}=\left(A F^{-1}[(\alpha\right.(1-\lambda)(A+B q)+(1-\alpha) \\
&\left.\times\left(A-\lambda(A+B q) F\left(x_{2}^{*}\right)\right)\right) \\
&\left.\times((1-\lambda)(A+B q))^{-1}\right] \\
&\left.+B q F^{-1}\left[\frac{(1-\alpha)\left(A-\lambda(A+B q) F\left(x_{2}^{*}\right)\right)}{(1-\lambda)(A+B q)}\right]\right) \\
& \times(A+B q)^{-1} .
\end{aligned}
$$

In fact, Problems $(\mathrm{P})$ and $\left(\mathrm{P}_{1}\right)$ can be seen as the special cases of Problem $\left(\mathrm{P}_{2}\right)$ when $\lambda=1$ and $\lambda=0$, respectively. By Theorem 11, we have the following conclusions.

(i) For $\lambda=1$, Problem $\left(\mathrm{P}_{2}\right)$ is reduced to problem (P). If it satisfies

$$
1=\lambda \geq \frac{A}{(A+B q) F\left[(A /(A+B q)) F^{-1}(\alpha)\right]},
$$

which implies that (44) holds, then the optimal solution to $\left(\mathrm{P}_{2}\right)$ is given by

$$
x_{2}^{*}=F^{-1}\left[\frac{A}{\lambda(A+B q)}\right] \text {. }
$$

It follows with $\lambda=1$ that

$$
x_{2}^{*}=F^{-1}\left[\frac{A}{\lambda(A+B q)}\right]=F^{-1}\left(\frac{A}{A+B q}\right) \text {, }
$$

which is the same as the optimal solution $x_{0}^{*}$ to $(\mathrm{P})$; otherwise, if it satisfies

$$
\lambda=1<\frac{A}{(A+B q) F\left[(A /(A+B q)) F^{-1}(\alpha)\right]},
$$

then the optimal solution $x_{2}^{*}$ to $\left(\mathrm{P}_{2}\right)$ is given by (46). By (58) in the proof of Theorem 11, $x_{2}^{*}$ solves

$$
\begin{aligned}
\frac{\partial k\left(x, v^{1}\right)}{\partial x}= & \lambda(A+B q) F(x)-A \\
& +\frac{1-\lambda}{1-\alpha}(A+B q) F\left(x-\frac{v^{1}}{B q}\right)=0
\end{aligned}
$$

which follows with $\lambda=1$ that

$$
\frac{\partial k\left(x, v^{1}\right)}{\partial x}=(A+B q) F(x)-A=0
$$




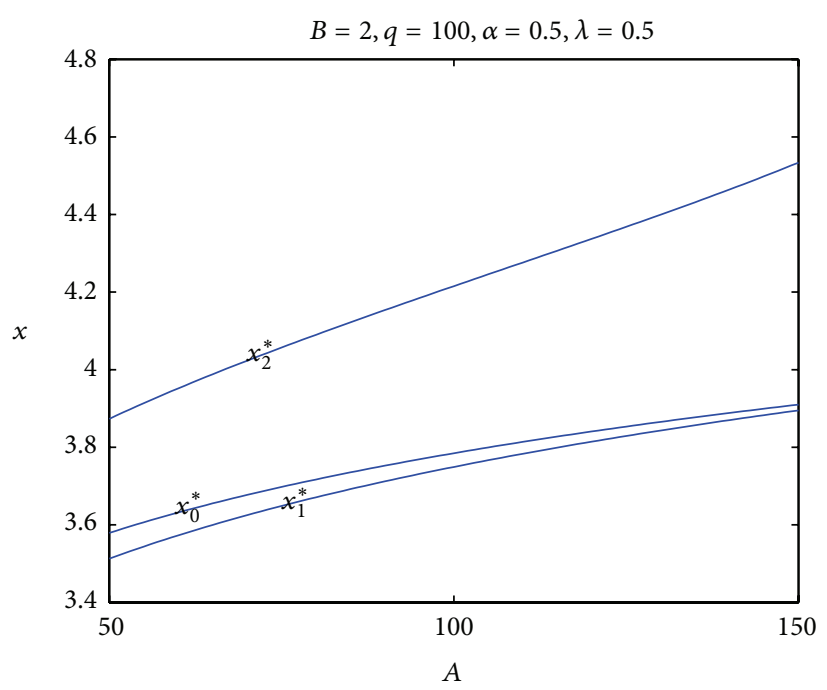

(a)

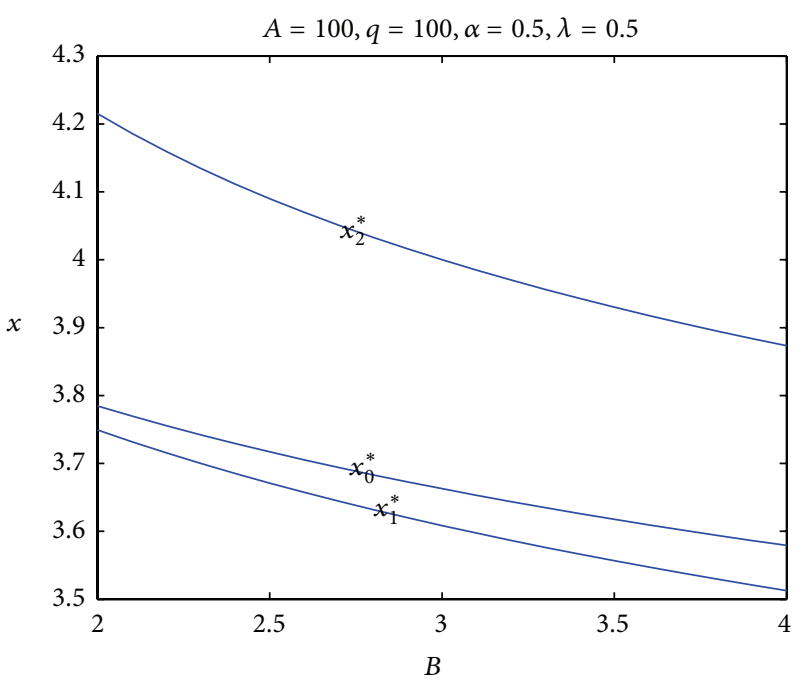

(b)

FIGURE 1: Optimal wholesale prices $x_{0}^{*}, x_{1}^{*}$, and $x_{2}^{*}$ for different shortage penalty coefficient $A$ and excess penalty coefficient $B$.

which implies

$$
x_{2}^{*}=F^{-1}\left(\frac{A}{A+B q}\right) \text {, }
$$

which is the same as the optimal solution $x_{0}^{*}$ to $(\mathrm{P})$, too.

(ii) For $\lambda=0$, Problem $\left(\mathrm{P}_{2}\right)$ is reduced to Problem $\left(\mathrm{P}_{1}\right)$. In this case, since

$$
\frac{A}{(A+B q) F\left[(A /(A+B q)) F^{-1}(\alpha)\right]}>0=\lambda,
$$

by Theorem 11, it is obvious that the optimal solution $x_{2}^{*}$ to $\left(\mathrm{P}_{2}\right)$ is given by

$$
\begin{gathered}
x_{2}^{*}=\left(\left[A F^{-1}((\alpha(1-\lambda)(A+B q)+(1-\alpha)\right.\right. \\
\left.\quad \times\left(A-\lambda(A+B q) F\left(x_{2}^{*}\right)\right)\right) \\
\left.\times((1-\lambda)(A+B q))^{-1}\right) \\
\left.\left.+B q F^{-1}\left(\frac{(1-\alpha)\left(A-\lambda(A+B q) F\left(x_{2}^{*}\right)\right)}{(1-\lambda)(A+B q)}\right)\right]\right) \\
\quad \times(A+B q)^{-1},
\end{gathered}
$$

which follows with $\lambda=0$ that

$$
x_{2}^{*}=\frac{1}{A+B q}\left[A F^{-1}\left(\frac{A+B q \alpha}{A+B q}\right)+B q F^{-1}\left(\frac{A(1-\alpha)}{A+B q}\right)\right] \text {, }
$$

Based on Corollaries 2-8, it is obvious that the optimal solution $x_{1}^{*}$ to $\left(\mathrm{P}_{2}\right)$ decreases with the growth of the excess penalty coefficient $B$ and the growth of the order quantity $q$ from the retailer and increases with the growth of shortage penalty coefficient $A$.

Remark 12. By Remark 9, the optimal solution to $\left(\mathrm{P}_{2}\right)$ may not be monotone with $\alpha$.

Remark 13. For the supplier, the optimal solution to $\left(\mathrm{P}_{2}\right)$ may not be monotone with $\lambda$. In fact, by Theorem 11, we have the following conclusion: if $\lambda \geq A /((A+B q) F[(A /(A+$ $\left.\left.B q)) F^{-1}(\alpha)\right]\right)$, we have $x_{2}^{*}=F^{-1}[A / \lambda(A+B q)]$, and it is obvious that $x_{2}^{*}$ decreases with the growth of $\lambda$; otherwise, $x_{2}^{*}$ is given by (46). Let

$$
\begin{gathered}
{\left[\frac{(1-\alpha)\left[A-\lambda(A+B q) F\left(x_{2}^{*}\right)\right]}{(1-\lambda)(A+B q)}\right]=H,} \\
\frac{\alpha(1-\lambda)(A+B q)+(1-\alpha)\left[A-\lambda(A+B q) F\left(x_{2}^{*}\right)\right]}{(1-\lambda)(A+B q)}=G .
\end{gathered}
$$

Then, we have

$$
x_{2}^{*}=\frac{A F^{-1}(G)+B q F^{-1}(H)}{A+B q},
$$

and it is obvious

which is the same as the optimal solution $x_{1}^{*}$ to $\left(\mathrm{P}_{1}\right)$.

$$
G=H+\alpha \geq H \text {. }
$$


Differentiating $x_{2}^{*}$ with respect to $\lambda$, we have

$$
\begin{aligned}
\frac{\partial x_{2}^{*}}{\lambda}\left[(A+B q)+\left(\frac{A}{f\left(F^{-1}(G)\right)}\right.\right. & \left.\left.+\frac{B q}{f\left(F^{-1}(H)\right)}\right) \frac{\lambda(1-\alpha) f\left(x_{2}^{*}\right)}{1-\lambda}\right] \\
= & {\left[\frac{A}{f\left(F^{-1}(G)\right)}+\frac{B q}{f\left(F^{-1}(H)\right)}\right] } \\
& \times \frac{(1-\alpha)\left[A-(A+B q) F\left(x_{2}^{*}\right)\right]}{(1-\lambda)^{2}(A+B q)} .
\end{aligned}
$$

The previous equality shows that the sign of $\partial x_{2}^{*} / \partial \lambda$ is the same as that of $A-(A+B q) F\left(x_{2}^{*}\right)$, which may be positive or negative.

\section{Numerical Example}

In this section, we will give an example to show the results obtained in Section 4.

Example 1. For a two-stage supply chain, suppose that the market wholesale price $\xi$ subjects to uniform distribution $U(3,5)$. Let us compute the optimal wholesale prices $x_{0}^{*}, x_{1}^{*}$, and $x_{2}^{*}$ for the supplier and give some sensitivity analysis.

Let $q=100, \alpha=0.5$, and $\lambda=0.5$, we compute the optimal wholesale prices $x_{0}^{*}, x_{1}^{*}$, and $x_{2}^{*}$ and illustrate the changes of these optimal wholesale prices with different parameters $A$ and $B$ in Figure 1 . By Figure 1 , it is easily checked that $x_{0}^{*}, x_{1}^{*}$, and $x_{2}^{*}$ are increasing in shortage penalty coefficient $A$ and decreasing in excess penalty coefficient $B$, respectively.

Moreover, for $A=100, B=2, q=100$, and $\lambda=0.5$, we compute the optimal wholesale prices $x_{1}^{*}$ and illustrate the changes of $x_{1}^{*}$ with confidence level $\alpha$ in Figure 2. By Figure 2, it is easily found that the optimal wholesale prices $x_{1}^{*}$ are decreasing in the confidence level $\alpha$.

\section{Conclusions}

With the growing emphasis on globalization, suppliers in two-stage supply chains encounter competitions from counterparts that provide the same products or services. A lower wholesale price certainly can attract customers, but it apparently reduces the profits of the suppliers. Thus, how to decide a wholesale price to coordinate/balance the two aspectsloss and risk-is very important. In this paper, we investigate the optimal pricing strategies of the suppliers in competitive circumstances. We introduce a new loss function for the suppliers, which considers the influence of wholesale prices to both the current loss and the future loss. Some optimal wholesale prices under different objectives are obtained. Further, the properties of these optimal wholesale prices are also studied. We think this paper provides some help for suppliers in deciding the wholesale price for their products.

Several extensions of this paper are possible. A further research is to consider the case where the order quantity of

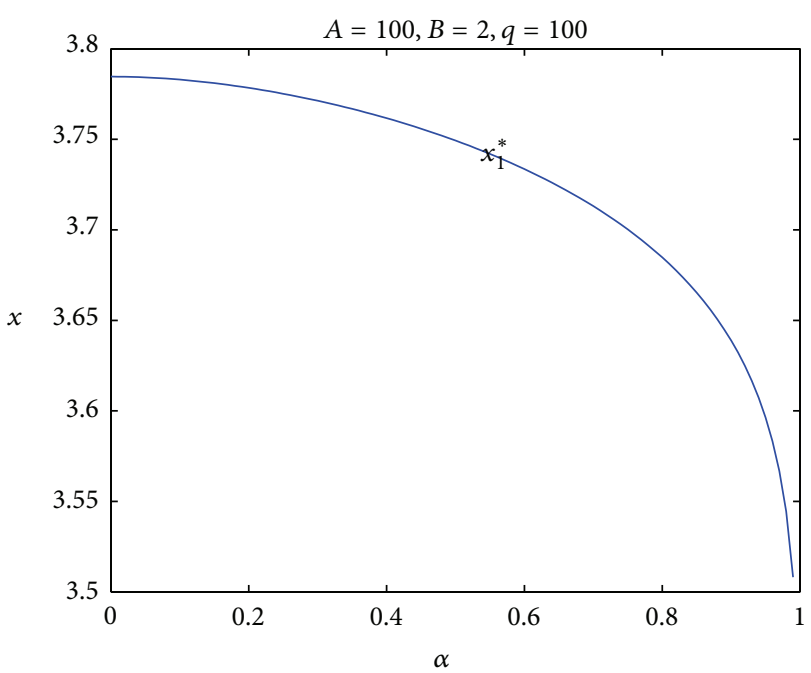

FIGURE 2: Optimal wholesale prices $x_{1}^{*}$ for different confidence level $\alpha$.

the retailer is wholesale price dependent. In such, the decision of an incumbent supplier as to wholesale price also influences the order quantity of the retailer, which has a more significant impact on his/her present and long-term profits.

\section{Acknowledgments}

This research is supported by the National Natural Science Foundation of China (71001089), the Natural Science Foundation of Zhejiang Province (Y13G010030) and the Science Foundation of Binzhou University (BZXYL1304).

\section{References}

[1] Y. Dai, X. Chao, S.-C. Fang, and H. L. W. Nuttle, "Pricing in revenue management for multiple firms competing for customers," International Journal of Production Economics, vol. 98, no. 1, pp. 1-16, 2005.

[2] S. Y. Sohn, T. H. Moon, and K. J. Seok, "Optimal pricing for mobile manufacturers in competitive market using genetic algorithm," Expert Systems with Applications, vol. 36, no. 2, pp. 3448-3453, 2009.

[3] T. Xiao and X. Qi, "Strategic wholesale pricing in a supply chain with a potential entrant," European Journal of Operational Research, vol. 202, no. 2, pp. 444-455, 2010.

[4] J. Li, S. Wang, and T. C. E. Cheng, "Competition and cooperation in a single-retailer two-supplier supply chain with supply disruption," International Journal of Production Economics, vol. 124, no. 1, pp. 137-150, 2010.

[5] J. Wang, A. Wang, and Y. Wang, "Markup pricing strategies between a dominant retailerand competitive manufacturers," Computers \& Industrial Engineering, vol. 64, no. 1, pp. 235-246, 2012.

[6] M. Voeth and U. Herbst, "Supply-chain pricing-a new perspective on pricing in industrial markets," Industrial Marketing Management, vol. 35, no. 1, pp. 83-90, 2006.

[7] F. J. Arcelus, S. Kumar, and G. Srinivasan, "Pricing and rebate policies in the two-echelon supply chain with asymmetric 
information under price-dependent, stochastic demand," International Journal of Production Economics, vol. 113, no. 2, pp. 598-618, 2008.

[8] J.-C. Lu, Y.-C. Tsao, and C. Charoensiriwath, "Competition under manufacturer service and retail price," Economic Modelling, vol. 28, no. 3, pp. 1256-1264, 2011.

[9] Y. Xia, "Competitive strategies and market segmentation for suppliers with substitutable products," European Journal of Operational Research, vol. 210, no. 2, pp. 194-203, 2011.

[10] X. Chen, L. Li, and M. Zhou, "Manufacturer's pricing strategy for supply chain with warranty period-dependent demand," Omega, vol. 40, no. 6, pp. 807-816, 2012.

[11] S. S. Sana, "Optimal contract strategies for two stage supply chain," Economic Modelling, vol. 30, pp. 253-260, 2013.

[12] P. Artzner, F. Delbaen, J.-M. Eber, and D. Heath, "Coherent measures of risk," Mathematical Finance, vol. 9, no. 3, pp. 203$228,1999$.

[13] H. Mauser and D. Rosen, "Beyond VaR: from measuring risk to managing risk," ALGO Research Quarterly, vol. 1, pp. 5-20, 1999.

[14] R. T. Rockafellar and S. Uryasev, "Optimization of conditional value-at-risk," The Journal of Risk, vol. 2, pp. 21-41, 2000.

[15] R. T. Rockafellar and S. Uryasev, "Conditional value-at-risk for general loss distributions," The Journal of Banking and Finance, vol. 26, no. 7, pp. 1443-1471, 2002. 


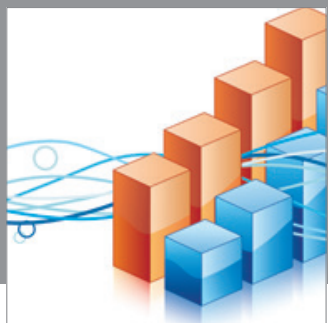

Advances in

Operations Research

mansans

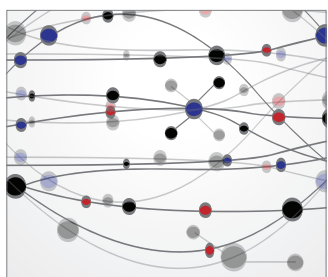

The Scientific World Journal
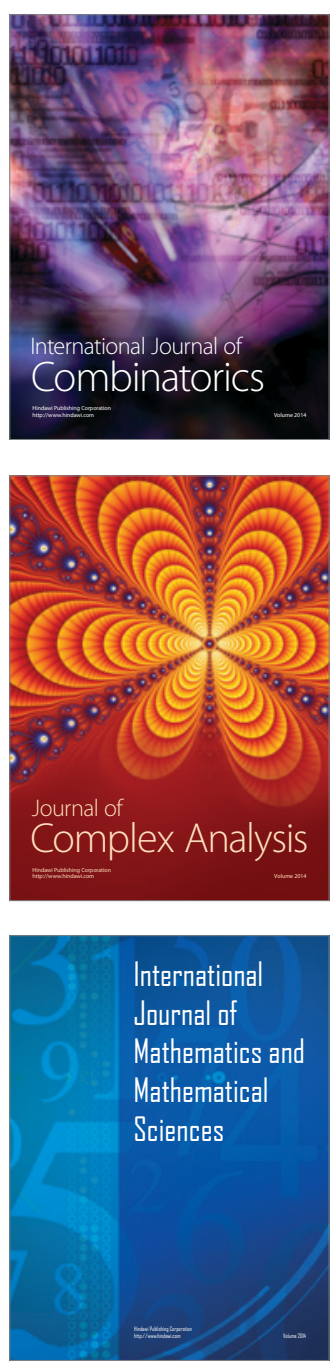
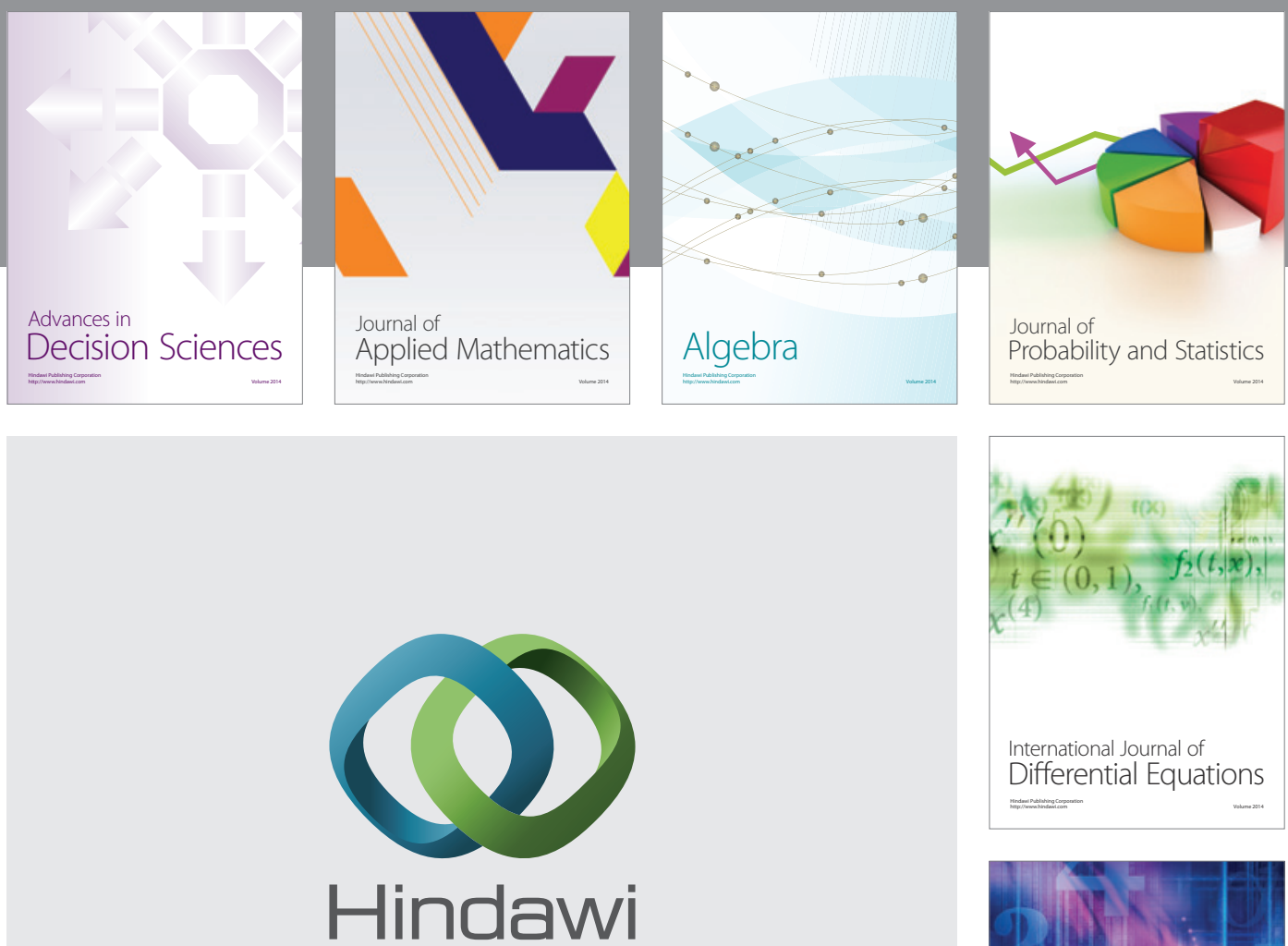

Submit your manuscripts at http://www.hindawi.com
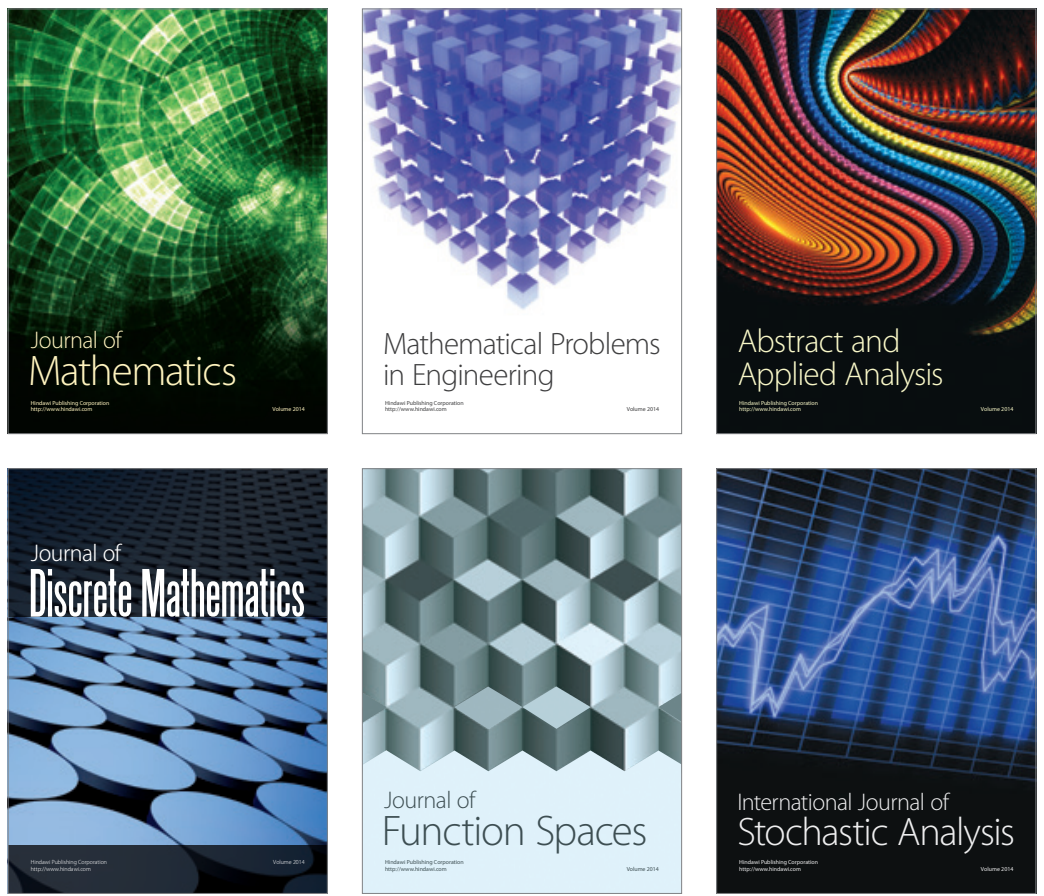

Journal of

Function Spaces

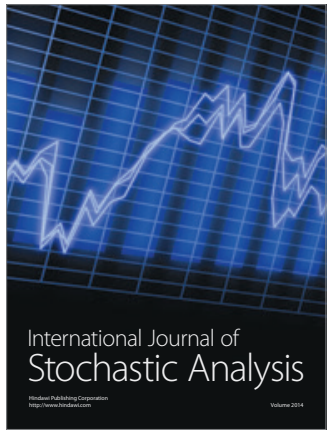

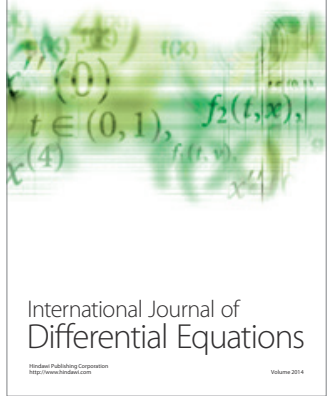
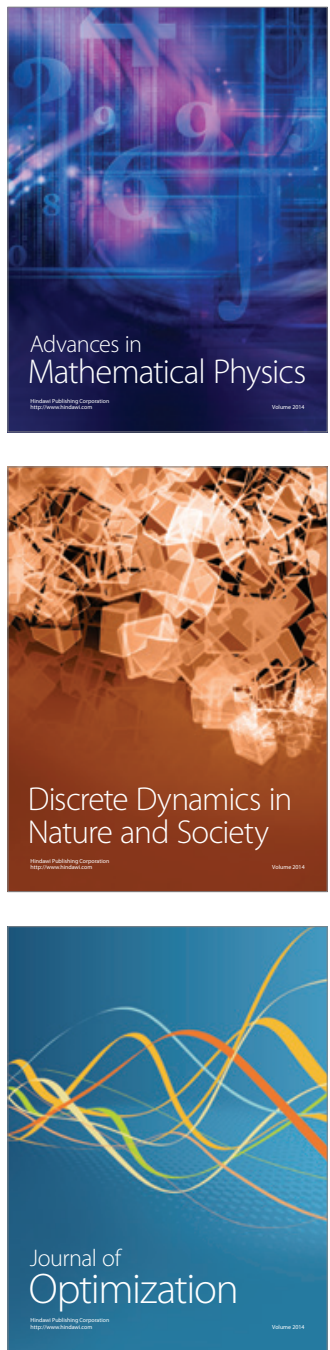